\title{
DIGITALCOMMONS
}

$11-1-2007$

\section{A Weighted Moving Average Process for Forcasting}

Shou Hsing Shih

University of South Florida, sshih@mail.usf.edu

Chris P. Tsokos

University of South Florida, profcpt@cas.usf.edu

Follow this and additional works at: http://digitalcommons.wayne.edu/jmasm

Part of the Applied Statistics Commons, Social and Behavioral Sciences Commons, and the Statistical Theory Commons

\section{Recommended Citation}

Shih, Shou Hsing and Tsokos, Chris P. (2007) "A Weighted Moving Average Process for Forcasting," Journal of Modern Applied Statistical Methods: Vol. 6 : Iss. 2 , Article 26.

DOI: $10.22237 /$ jmasm/1193891100

Available at: http://digitalcommons.wayne.edu/jmasm/vol6/iss2/26 


\title{
A Weighted Moving Average Process for Forecasting
}

\author{
Shou Hsing Shih Chris P. Tsokos \\ University of South Florida
}

A forecasting model for a nonstationary stochastic realization is proposed based on modifying a given time series into a new k-time moving average time series. The study is based on the autoregressive integrated moving average process along with its analytical constrains. The analytical procedure of the proposed model is given. A stock XYZ selected from the Fortune 500 list of companies and its daily closing price constitute the time series. Both the classical and proposed forecasting models were developed and a comparison of the accuracy of their responses is given.

Key words: ARIMA, moving average, stock, time series analysis

Introduction

Time series analysis and modeling plays a very important role in forecasting, especially when our initial stochastic realization is nonstationary in nature. Some of the interesting and useful publications related to the subject area are Akaike (1974), Banerjee et al. (1993), Box et al. (1994), Brockwell and Davis (1996), Dickey and Fuller (1979), Dickey et al. (1984), Durbin and Koopman (2001), Gardner et al. (1980), Harvey (1993), Jones (1980), Kwiatkowski et al. (1992), Rogers (1986), Said and Dickey (1984), Sakamoto et al. (1986), Shumway and Stoffer (2006), Tsokos (1973), Wei (2006).

The purpose of this study is to begin with a given time series that characterizes an economic or any other natural phenomenon and as usual, is nonstationary. Box and Jenkins (1994) developed a popular and useful classical procedure to develop forecasting models that have been shown to be effective. In this article,

Shou Hsing Shih recently received the Ph. D. in Statistics from University of South Florida. Shih's research mainly concentrates on time series forecasting. E-mail address: sshih3@tampabay.rr.com. Chris P. Tsokos is Distinguished University Professor of Mathematics and Statistics at the University of South Florida. He is the author of more than 250 research publications. E-mail address: profcpt@cas.usf.edu a procedure for developing a forecasting model that is more effective than the classical approach is introduced. For a given stationary or nonstationary time series, $\left\{x_{t}\right\}$, generate a k-day moving average time series, $\left\{y_{t}\right\}$, and the developmental process begins.

Certain basic concepts and analytical methods are reviewed that are essential in structuring the proposed forecasting model. The review is based on the autoregressive integrated moving average processes. The accuracy of the proposed forecasting model is illustrated by selecting from the list of Fortune 500 companies, company $\mathrm{XYZ}$, and considering its daily closing prices for 500 days. The classical time series model for the subject information along with the proposed process was developed. A statistical comparison based on the actual and forecasting residuals is given, both in tabular and graphical form.

The Proposed Forecasting Model: k-th Moving Average

It is not appropriate to build a time series model without conforming to certain mathematical constrains, such as stationarity of a given stochastic realization. Almost always, the time series that is given is nonstationary in nature and then, the next step is to reduce it into being stationary. Let $\left\{x_{t}\right\}$ be the original time series. The difference filter is given by 


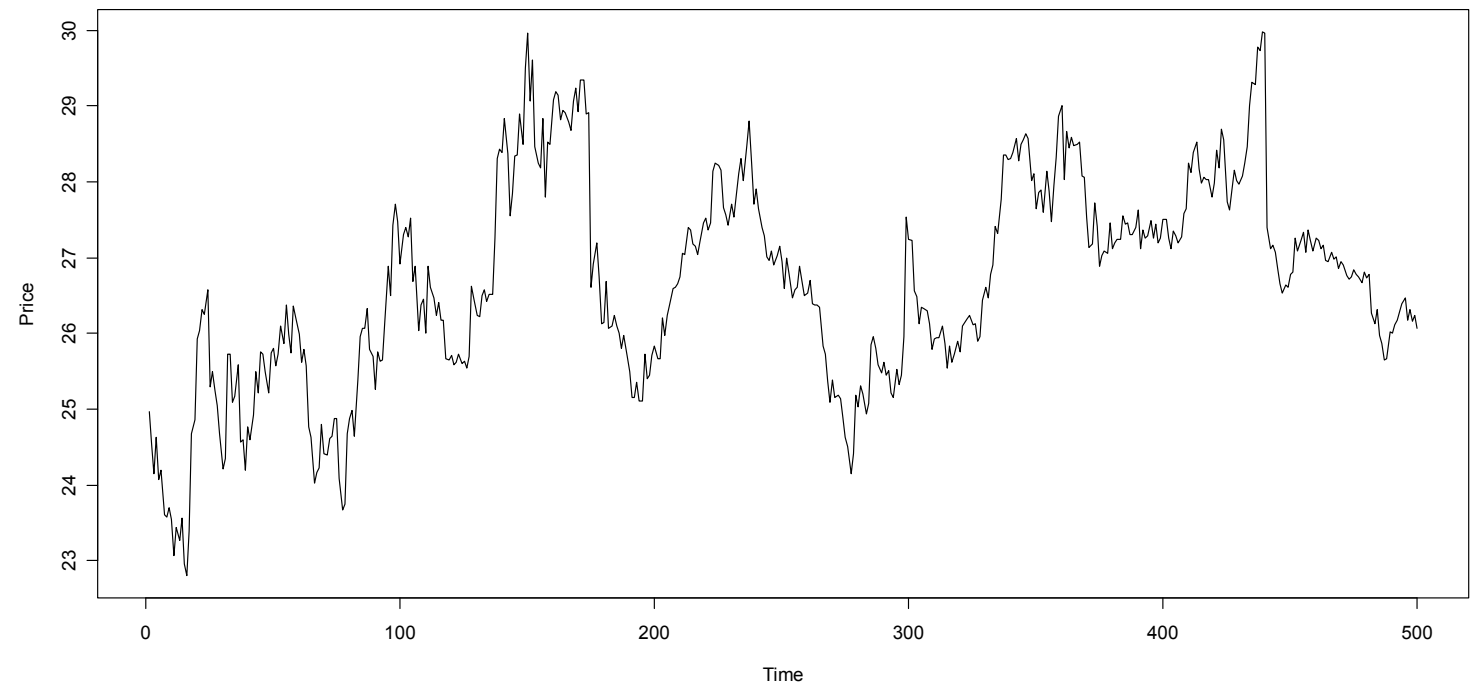

Figure 1. Daily Closing Price for Stock XYZ

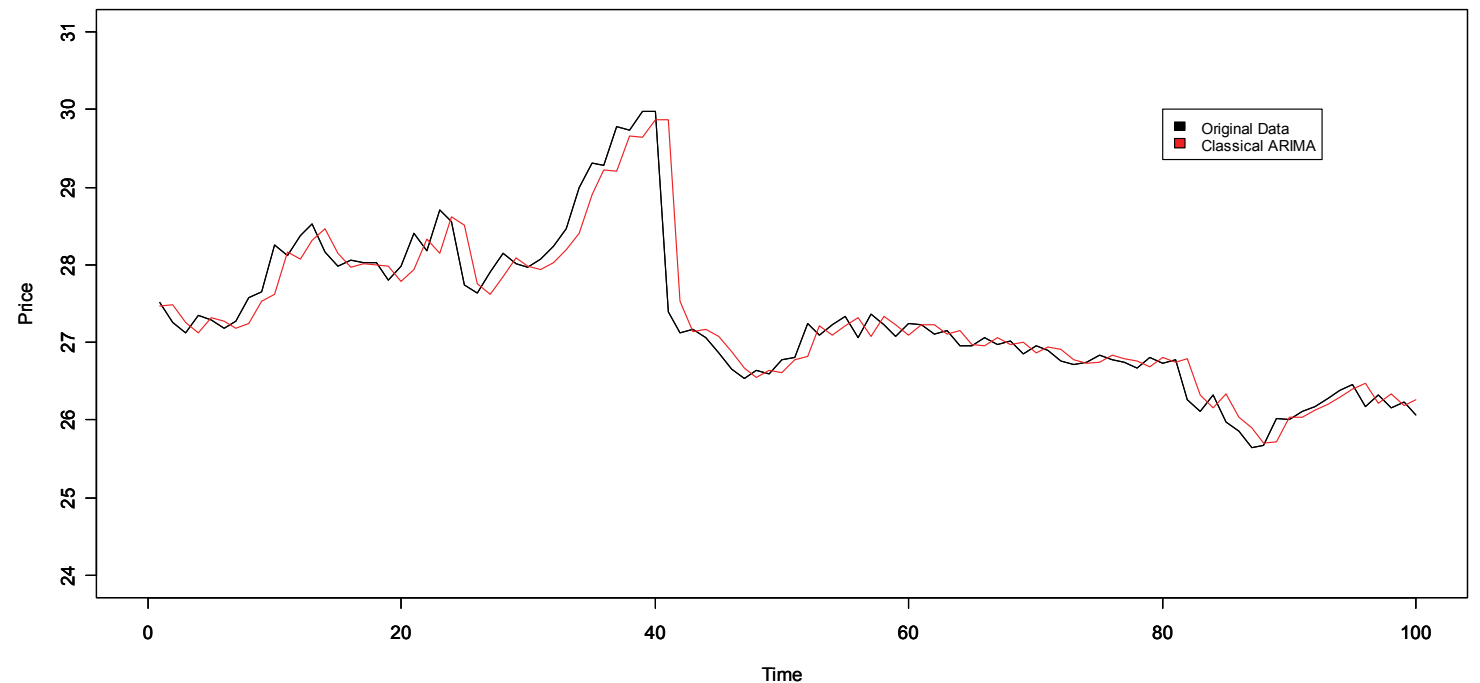

Figure 2. Comparisons on Classical ARIMA Model VS. Original Time Series for the Last 100 Observations 


$$
(1-B)^{d}
$$

where $B^{j} x_{t}=x_{t-j}$, and $\mathrm{d}$ is the degree of differencing of the series.

The primary use for the k-th moving average process is for smoothing a realized time series. It is very useful in discovering a shortterm, long-term trends and seasonal components of a given time series. The $\mathrm{k}$-th moving average process of a time series $\left\{x_{t}\right\}$ is defined as follows:

$$
y_{t}=\frac{1}{k} \sum_{j=0}^{k-1} x_{t-k+1+j}
$$

where $t=k, k+1, \ldots, n$.

As $\mathrm{k}$ increases, the number of observations $\mathrm{k}$ of $\left\{y_{t}\right\}$ decreases, and $\left\{y_{t}\right\}$ gets closer to the mean of $\left\{x_{t}\right\}$ as $\mathrm{k}$ increases. When $k=n,\left\{y_{t}\right\}$ reduces to only a single observation, and equals $\mu$, that is

$$
y_{t}=\frac{1}{n} \sum_{j=1}^{n} x_{j}=\mu
$$

Then, develop the proposed model by transforming the original time series $\left\{x_{t}\right\}$ into $\left\{y_{t}\right\}$ by applying (2). After establishing the new time series, usually nonstationary, begin the process of reducing it into a stationary time series. Kwiatkowski, et al. (1992) introduced the KPSS Test to check the level of stationarity of a time series. Apply the differencing order $\mathrm{d}$ to the new time series $\left\{y_{t}\right\}$ for $d=0,1,2, \ldots$, then verify the stationarity of the series with the KPSS test until the series become stationary. Therefore, one can reduce the nonstationary time series into a stationary one after a proper number of differencing. Then proceed the model building procedure of developing the proposed forecasting model.
After choosing a proper degree of differencing $\mathrm{d}$, proceed with the model building process by assuming different orders for the autoregressive integrated moving average model, ARIMA(p,d,q), also known as Box and Jenkins method, where $(p, d, q)$ represent the order of the autoregressive process, the order of differencing and the order of the moving average process, respectively. The $\operatorname{ARIMA}(\mathrm{p}, \mathrm{d}, \mathrm{q})$ is defined as:

$$
\phi_{p}(B)(1-B)^{d} y_{t}=\theta_{q}(B) \varepsilon_{t}
$$

where $\left\{y_{t}\right\}$ is the realized time series, $\phi_{p}$ and $\theta_{q}$ are the weights or coefficients of the AR and MA that drive the model, respectively, and $\mathcal{E}_{t}$ is the random error. Write $\phi_{p}$ and $\theta_{q}$ as

$$
\begin{gathered}
\phi_{p}(B)= \\
\left(1-\phi_{1} B-\phi_{2} B^{2}-\ldots-\phi_{p} B^{p}\right)
\end{gathered}
$$

and

$$
\begin{gathered}
\theta_{q}(B)= \\
\left(1-\theta_{1} B-\theta_{2} B^{2}-\ldots-\theta_{q} B^{q}\right)
\end{gathered}
$$

Sometimes it is difficult to make a decision in selecting the best order of the $\operatorname{ARIMA}(\mathrm{p}, \mathrm{d}, \mathrm{q})$ model when there are several models that all adequately represent a given set of time series. Hence, Akaile's information criterion (AIC) (1974), plays a major role when it comes to model selection. AIC was introduced by Akaike in 1973, and it is defined as:

$$
\operatorname{AIC}(M)=
$$

$$
-2 \ln \text { [maximum likelihood] }+2 M \text {, }
$$

where $\mathrm{M}$ is the number of parameters in the model and the unconditional log-likelihood function suggested by Box, Jenkins, and Reinsel (1994), is given by

$$
\ln L\left(\phi, \mu, \theta, \sigma_{\varepsilon}^{2}\right)=
$$


Table 1. Basic Evaluation Statistics
$\bar{r}$

$$
S_{r}^{2}
$$
$S_{r}$

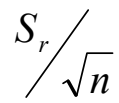
0.02209169
0.1445187
0.3801562
0.0170011

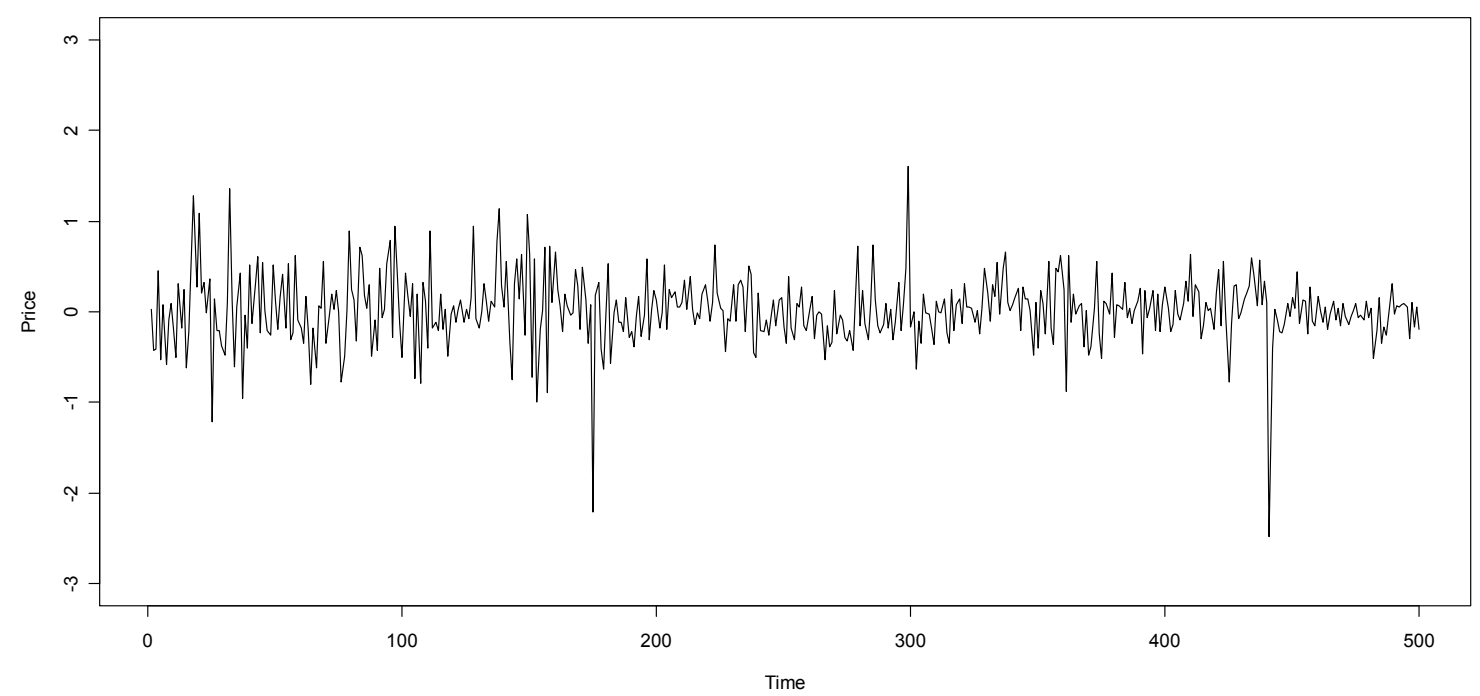

Figure 3. Time Series Plot of the Residuals for Classical Model

$$
-\frac{n}{2} \ln 2 \pi \sigma_{\varepsilon}^{2}-\frac{S(\phi, \mu, \theta)}{2 \sigma_{\varepsilon}^{2}}
$$

where $S(\phi, \mu, \theta)$ is the unconditional sum of squares function given by

$$
\begin{gathered}
S(\phi, \mu, \theta)= \\
\sum_{t=-\infty}^{n}\left[E\left(\varepsilon_{t} \mid \phi, \mu, \theta, y\right)\right]^{2}
\end{gathered}
$$

where $E\left(\varepsilon_{t} \mid \phi, \mu, \theta, y\right)$ is the conditional expectation of $\varepsilon_{t}$ given $\phi, \mu, \theta, y$.
The quantities $\hat{\phi}, \hat{\mu}$, and $\hat{\theta}$ that maximize (8) are called unconditional maximum likelihood estimators. Because $\ln L\left(\phi, \mu, \theta, \sigma_{\varepsilon}^{2}\right)$ involves the data only through $S(\phi, \mu, \theta)$, these unconditional maximum likelihood estimators are equivalent to the unconditional least squares estimators obtained by minimizing $S(\phi, \mu, \theta)$. In practice, the summation in (9) is approximated by a finite form

$$
\begin{gathered}
S(\phi, \mu, \theta)= \\
\sum_{t=M}^{n}\left[E\left(\varepsilon_{t} \mid \phi, \mu, \theta, y\right)\right]^{2}
\end{gathered}
$$




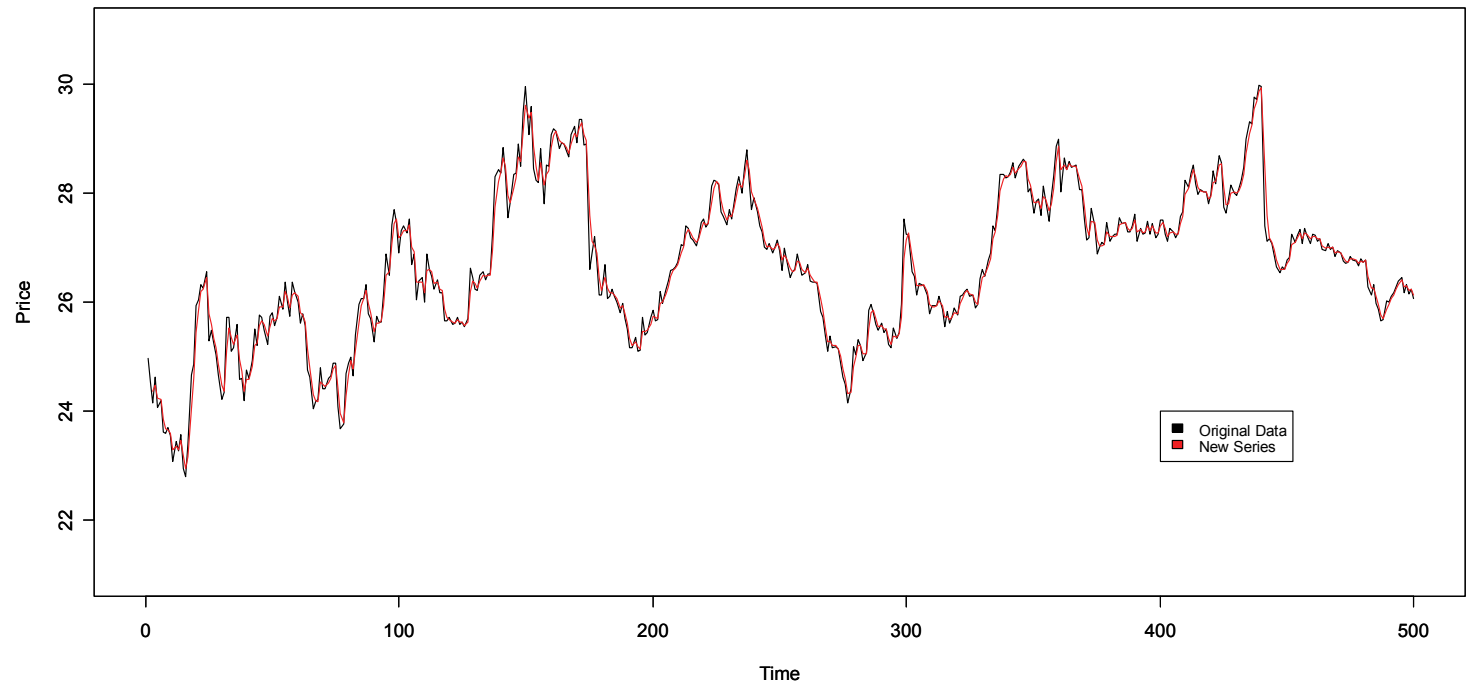

Figure 4. Three Days Moving Average on Daily Closing Price of Stock XYZ Vs. the original time series

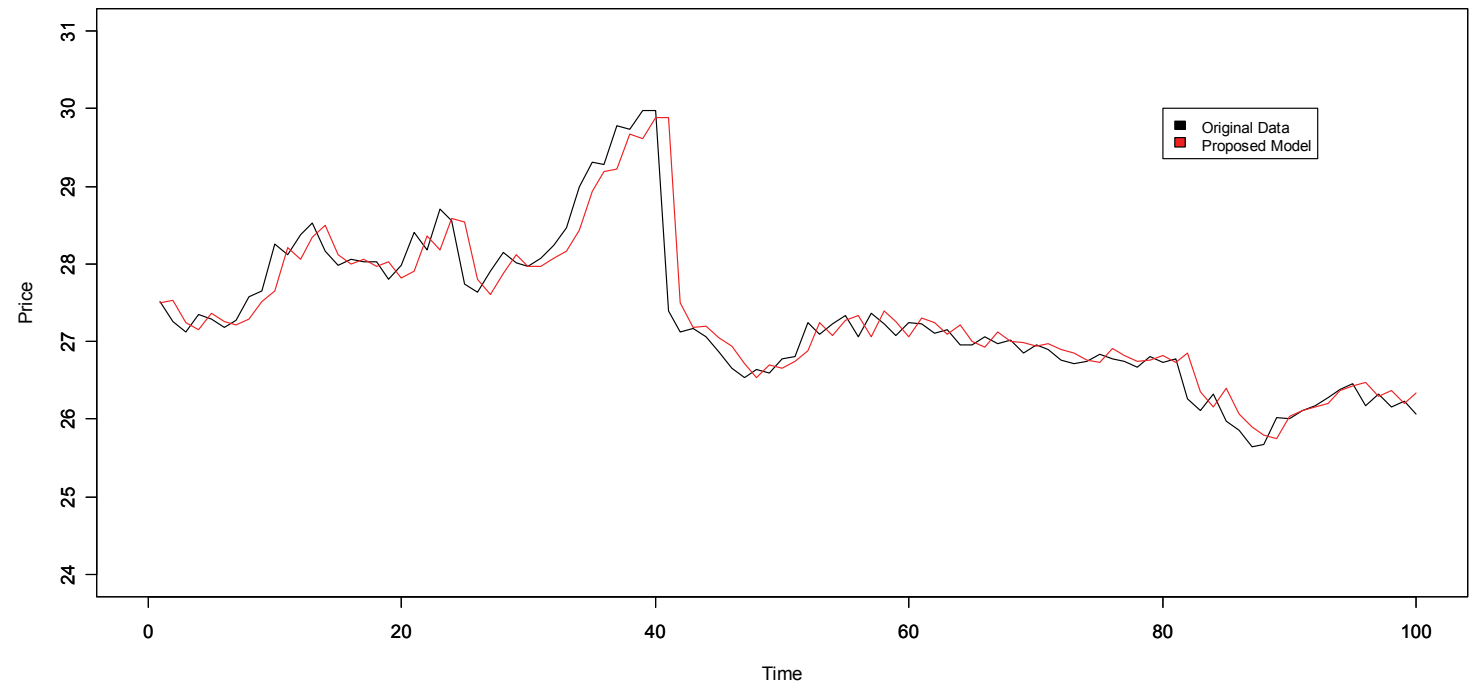

Figure 5. Comparisons on Our Proposed Model VS. Original Time Series for the Last 100 Observations 


\begin{tabular}{cccc}
\multicolumn{4}{c}{ Table 2. Actual and Predicted Price } \\
$\mathrm{N}$ & Actual Price & Predicted Price & Residuals \\
476 & 26.78 & 26.8473 & -0.0673 \\
477 & 26.75 & 26.7976 & -0.0476 \\
478 & 26.67 & 26.7673 & -0.0972 \\
479 & 26.8 & 26.6922 & 0.1078 \\
480 & 26.73 & 26.8064 & -0.0764 \\
481 & 26.78 & 26.7490 & 0.0310 \\
482 & 26.27 & 26.7911 & -0.5211 \\
483 & 26.12 & 26.3277 & -0.2077 \\
484 & 26.32 & 26.1631 & 0.1569 \\
485 & 25.98 & 26.3364 & -0.3564 \\
486 & 25.86 & 26.0349 & -0.1749 \\
487 & 25.65 & 25.9068 & -0.2568 \\
488 & 25.67 & 25.6670 & 0.0031 \\
489 & 26.02 & 25.7119 & 0.3081 \\
490 & 26.01 & 26.0335 & -0.0235 \\
491 & 26.11 & 26.0427 & 0.0674 \\
492 & 26.18 & 26.1343 & 0.0457 \\
493 & 26.28 & 26.2032 & 0.0768 \\
494 & 26.39 & 26.2986 & 0.0914 \\
495 & 26.46 & 26.4043 & 0.0557 \\
496 & 26.18 & 26.4743 & -0.2943 \\
497 & 26.32 & 26.2219 & 0.0981 \\
498 & 26.16 & 26.3354 & -0.1754 \\
499 & 26.24 & 26.1953 & 0.0447 \\
500 & 26.07 & 26.2602 & -0.1902
\end{tabular}

where $\mathrm{M}$ is a sufficiently large integer such that the backcast increment $\left|E\left(\varepsilon_{t} \mid \phi, \mu, \theta, y\right)-E\left(\varepsilon_{t-1} \mid \phi, \mu, \theta, y\right)\right|$ is less than any arbitrary predetermined small $\mathcal{E}$ value for $t \leq-(M+1)$. This expression implies that $E\left(\varepsilon_{t} \mid \phi, \mu, \theta, y\right) \cong \mu$; hence, $E\left(\varepsilon_{t} \mid \phi, \mu, \theta, y\right)$ is negligible for $t \leq-(M+1)$.

After obtaining the parameter estimates $\hat{\phi}, \hat{\mu}$, and $\hat{\theta}$, the estimate $\hat{\sigma}_{\varepsilon}^{2}$ of $\sigma_{\varepsilon}^{2}$ can then be calculated from

$$
\hat{\sigma}_{\varepsilon}^{2}=\frac{S(\hat{\phi}, \hat{\mu}, \hat{\theta})}{n}
$$

For an $\operatorname{ARMA}(\mathrm{p}, \mathrm{q})$ model based on $\mathrm{n}$ observations, the log-likelihood function is

$$
\begin{gathered}
\ln L= \\
-\frac{n}{2} \ln 2 \pi \sigma_{\varepsilon}^{2}-\frac{1}{2 \sigma_{\varepsilon}^{2}} S(\phi, \mu, \theta)
\end{gathered}
$$

Proceed to maximize (12) with respect to the parameters $\phi, \mu, \theta$, and $\sigma_{\varepsilon}^{2}$, from (11),

$$
\ln \hat{L}=
$$




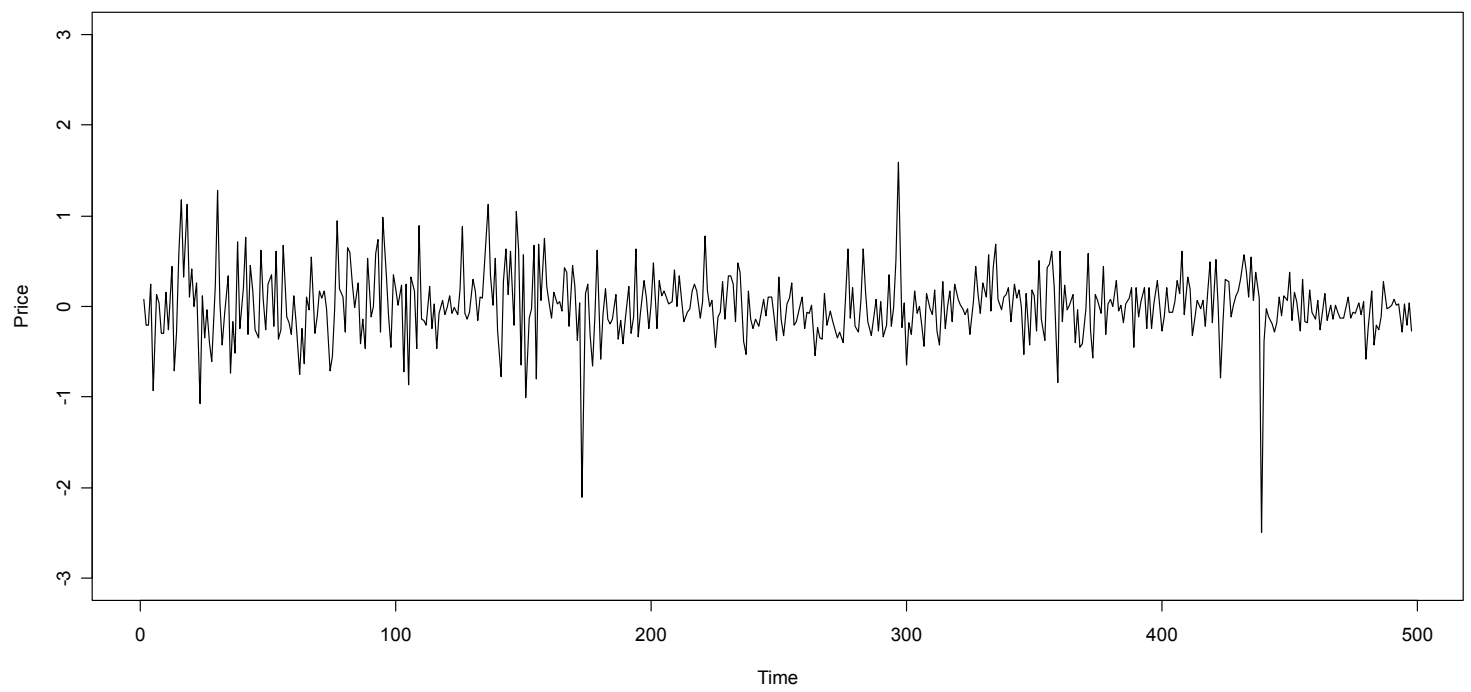

Figure 6. Time Series Plot for Residuals for Our Proposed Model

Table 3. Basic Evaluation Statistics

$\begin{array}{cccc}\bar{r} & S_{r}^{2} & S_{r} & S_{r} / \sqrt{n} \\ 0.01016814 & 0.1437259 & 0.3791119 & 0.01698841\end{array}$

$$
-\frac{n}{2} \ln \hat{\sigma}_{\varepsilon}^{2}-\frac{n}{2}(1+\ln 2 \pi)
$$

Because the second term in expression (13) is a constant, reduce the AIC to

$$
\operatorname{AIC}(M)=n \ln \hat{\sigma}_{\varepsilon}^{2}+2 M
$$

Thus, we an appropriate time series model is generated and the statistical process with the smallest AIC can be selected. The model identified will possess the smallest average mean square error. The development of the model is summarized as follows.

Transform the original time series $\left\{x_{t}\right\}$ into a new series $\left\{y_{t}\right\}$.

- Check for stationarity of the new time
- series $\left\{y_{t}\right\}$ by determining the order of differencing $\mathrm{d}$, where $d=0,1,2, \ldots$ according to KPSS test, until stationarity is achieved.

- Decide the order $m$ of the process, for this case, let $m=5$ where $p+q=m$.

- $\operatorname{After}(\mathrm{d}, m)$ is selected, list all possible set of $(\mathrm{p}, \mathrm{q})$ for $p+q \leq m$.

- For each set of (p, q), estimate the parameters of each model, that is, $\phi_{1}, \phi_{2}, \ldots, \phi_{p}, \theta_{1}, \theta_{2}, \ldots, \theta_{q}$

- Compute the AIC for each model, and choose the one with smallest AIC. 
Table 4. Actual and Predicted Price

$\begin{array}{cccc}\text { N } & \text { Actual Price } & \text { Predicted Price } & \text { Residuals } \\ 476 & 26.78 & 26.8931 & -0.1131 \\ 477 & 26.75 & 26.7715 & -0.0215 \\ 478 & 26.67 & 26.7121 & -0.0421 \\ 479 & 26.8 & 26.7239 & 0.0761 \\ 480 & 26.73 & 26.7854 & -0.0554 \\ 481 & 26.78 & 26.6892 & 0.0908 \\ 482 & 26.27 & 26.8292 & -0.5592 \\ 483 & 26.12 & 26.3027 & -0.1827 \\ 484 & 26.32 & 26.0808 & 0.2392 \\ 485 & 25.98 & 26.3603 & -0.3803 \\ 486 & 25.86 & 25.9868 & -0.1268 \\ 487 & 25.65 & 25.8443 & -0.1943 \\ 488 & 25.67 & 25.7115 & -0.0414 \\ 489 & 26.02 & 25.6499 & 0.3701 \\ 490 & 26.01 & 25.9650 & 0.0450 \\ 491 & 26.11 & 26.0526 & 0.0574 \\ 492 & 26.18 & 26.0912 & 0.0888 \\ 493 & 26.28 & 26.1449 & 0.1351 \\ 494 & 26.39 & 26.3090 & 0.0810 \\ 495 & 26.46 & 26.3752 & 0.0848 \\ 496 & 26.18 & 26.4223 & -0.2423 \\ 497 & 26.32 & 26.2461 & 0.0739 \\ 498 & 26.16 & 26.2964 & -0.1364 \\ 499 & 26.24 & 26.1437 & 0.0963 \\ 500 & 26.07 & 26.2678 & -0.1978\end{array}$

According to the criterion mentioned above, the ARIMA $(p, d, q)$ model can be obtained that best fit a given time series, where the coefficients are $\phi_{1}, \phi_{2}, \ldots, \phi_{p}, \theta_{1}, \theta_{2}, \ldots, \theta_{q}$. Using the model that we developed for $\left\{y_{t}\right\}$ and subject to the AIC criteria, we forecast values of $\left\{y_{t}\right\}$ and proceed to apply the back-shift operator to obtain estimates of the original phenomenon $\left\{x_{t}\right\}$, that is,

$$
\begin{aligned}
& \hat{x_{t}}= \\
& k \hat{y_{t}}-x_{t-1}-x_{t-2}-\ldots-x_{t-k+1}
\end{aligned}
$$

The proposed model and the corresponding procedure discussed in this section shall be illustrated with real economic application and the results will be compared with the classical time series model.

The proposed model and the corresponding procedure discussed in this section shall be illustrated with real economic application and the results will be compared with the classical time series model. 
Table 5. Basic Comparison on Classical Approach Vs. Our Proposed model

$\begin{array}{ccccc} & \bar{r} & S_{r}^{2} & S_{r} & S_{r} / \sqrt{n} \\ \text { Classical } & 0.02209169 & 0.1445187 & 0.3801562 & 0.0170011 \\ & & & & \\ \text { Proposed } & 0.01016814 & 0.1437259 & 0.3791119 & 0.01698841\end{array}$

First, a time series forecasting model is developed of the given nonstationary data using the ordinary Box and Jenkins methodology. Secondly, the data are modified, Figure 1, to develop the proposed time series forecasting model. A comparison of the two models will be given.

The general theoretical form of the $\operatorname{ARIMA}(\mathrm{p}, \mathrm{d}, \mathrm{q})$ is given by

$$
\phi_{p}(B)(1-B)^{d} x_{t}=\theta_{q}(B) \varepsilon_{t}
$$

Following the Box and Jenkins' methodology (1994), the classical forecasting model with the best AIC score is the $\operatorname{ARIMA}(1,1,2)$. That is, a combination of first order autoregressive (AR) and a second order moving average (MA) with a first difference filter. Write it as

$$
\begin{gathered}
(1-.9631 B)(1-B) x_{t}= \\
\left(1-1.0531 B+.0581 B^{2}\right) \varepsilon_{t}
\end{gathered}
$$

After expanding the autoregressive operator and the difference filter,

$$
\begin{aligned}
& \left(1-1.9631 B+.9631 B^{2}\right) x_{t}= \\
& \left(1-1.0531 B+.0581 B^{2}\right) \varepsilon_{t}
\end{aligned}
$$

and rewrite the model as

$$
\begin{aligned}
& x_{t}=1.9631 x_{t-1}-.9631 x_{t-2}+ \\
& \varepsilon_{t}-1.0531 \varepsilon_{t-1}+.0581 \varepsilon_{t-2}
\end{aligned}
$$

by letting $\varepsilon_{t}=0$, there is the one day ahead forecasting time series of the closing price of stock XYZ as

$$
\begin{aligned}
& \hat{x}_{t}=1.9631 x_{t-1}-.9631 x_{t-2} \\
& -1.0531 \varepsilon_{t-1}+.0581 \varepsilon_{t-2}
\end{aligned}
$$

Using the above equation, graph the forecasting values obtained by using the classical approach on top of the original time series, as shown by Figure 2.

The basic statistics that reflect the accuracy of model (20) are the mean $\bar{r}$, variance $S_{r}^{2}$, standard deviation $S_{r}$ and standard error $S_{r} / \sqrt{n}$ the $n$ of the residuals. Figure 3 gives a plot of the residual and Table 1 gives the basic statistics.

Furthermore, restructure the model (20) with $n=475$ data points to forecast the last 25 observations only using the previous information. The purpose is to see how accurate our forecast prices are with respect to the actual 25 values that have not been used. Table 2 gives the actual price, predicted price, and residuals between the forecasts and the 25 hidden values.

The average of these residuals is $\bar{r}=-0.05608$. Proceed to develop the proposed forecasting model. The original time series of stock XYZ daily closing prices is given by Figure 1 . The new time series is being created by $k=3$ days moving average and the analytical form of $\left\{y_{t}\right\}$ is given by 


$$
y_{t}=\frac{x_{t-2}+x_{t-1}+x_{t}}{3}
$$

Figure 4 shows the new time series $\left\{y_{t}\right\}$ along with the original time series $\left\{x_{t}\right\}$, that will be used to develop the proposed forecasting model. The best model that characterizes the behavior of $\left\{y_{t}\right\}$ is $\operatorname{ARIMA}(2,1,3)$. That is,

$$
\begin{aligned}
& \left(1-.8961 B-.0605 B^{2}\right)(1-B) y_{t}= \\
& \left(1+.0056 B-.0056 B^{2}-B^{3}\right) \varepsilon_{t}
\end{aligned}
$$

Expanding the autoregressive operator and the first difference filter, we have

$$
\begin{aligned}
& \left(1-1.8961 B+.8356 B^{2}+.0605 B^{3}\right) y_{t}= \\
& \left(1+.0056 B-.0056 B^{2}-B^{3}\right) \varepsilon_{t}
\end{aligned}
$$

Thus, write (23) as

$$
\begin{gathered}
y_{t}= \\
1.8961 y_{t-1}-.8356 y_{t-2}-.0605 y_{t-3} \\
+\varepsilon_{t}+.0056 \varepsilon_{t-1}-.0056 \varepsilon_{t-2}-\varepsilon_{t-3}
\end{gathered}
$$

The final analytical form of the proposed forecasting model can be written as

$$
\begin{gathered}
\hat{y_{t}}= \\
1.8961 y_{t-1}-.8356 y_{t-2}-.0605 y_{t-3} \\
+.0056 \varepsilon_{t-1}-.0056 \varepsilon_{t-2}-\varepsilon_{t-3}
\end{gathered}
$$

Using the above equation, a plot of the developed model (25), showing a one day ahead forecasting along with the new time series, $\left\{y_{t}\right\}$, is displayed by Figure 5 .

Note the closeness of the two plots that reflect the quality of the proposed model.

Similar to the classical model approach that we discussed earlier, use the first 475 observations $\left\{y_{1}, y_{2}, \ldots, y_{475}\right\}$ to forecast $\hat{y_{476}}$. Then, use the observations $\left\{y_{1}, y_{2}, \ldots, y_{476}\right\}$ to forecast $\hat{y_{477}}$, and continue this process until forecasts are obtained for all the observations, that is, $\left\{\hat{y_{476}}, \hat{y_{477}}, \ldots, \hat{y_{500}}\right\}$. From equation (21), the relationship can be seen between the forecasting values of the original series $\left\{x_{t}\right\}$ and the forecasting values of 3 days moving average series $\left\{y_{t}\right\}$, that is,

$$
\hat{x_{t}}=3 \hat{y_{t}}-x_{t-1}-x_{t-2}
$$

Hence, after $\left\{\hat{y_{476}}, \hat{y_{477}}, \ldots, \hat{y_{500}}\right\}$ is estimated, use the above equation, (26), to solve the forecasting values for $\left\{x_{t}\right\}$. Figure 6 is the residual plot generated by the proposed model, and followed by Table 3, that includes the basic evaluation statistics.

Both of the above displayed evaluations reflect on accuracy of the proposed model. The actual daily closing prices of stock XYZ from the 476th day along with the forecasted prices and residuals are given in Table 4 . The results given above attest to the good forecasting estimates for the hidden data.

Comparison of the Forecasting Models

In this section, the two developed models are compared. The classical process is given by

$$
\begin{gathered}
\hat{x_{t}}=1.9631 x_{t-1}-.9631 x_{t-2}- \\
1.0531 \varepsilon_{t-1}+.0581 \varepsilon_{t-2}
\end{gathered}
$$

In the proposed model, the following inversion is used to obtain the estimated daily closing prices of stock $\mathrm{XYZ}$, that is,

$$
\begin{gathered}
\hat{y_{t}}= \\
1.8961 y_{t-1}-.8356 y_{t-2}-.0605 y_{t-3} \\
+.0056 \varepsilon_{t-1}-.0056 \varepsilon_{t-2}-\varepsilon_{t-3}
\end{gathered}
$$

in conjunction with 
$\hat{x}_{t}=3 \hat{y_{t}}-x_{t-1}-x_{t-2}$

Table 5 is a comparison of the basic statistics used to evaluate the two models under investigation. The average mean residuals between the two models shown that the proposed model is overall approximately 54\% more effective in estimating one day ahead the closing price of Fortune 500 stock XYZ.

\section{Conclusion}

Based on the average mean residuals the proposed model was significantly more effective in such term of predicting of the closing daily prices of stock XYZ.

\section{References}

Akaike, H. (1974). A New Look at the Statistical Model Identification, IEEE Transactions on Automatic Control, AC-19, 716-723.

Banerjee, A., Dolado, J. J., Galbraith, J. W., \& Hendry, D. F. (1993). Cointegration, Error Correction, and the Econometric Analysis of Non-Stationary Data, Oxford University Press, Oxford.

Box, G. E. P., Jenkins, G. M., \& Reinsel, G. C. (1994). Time Series Analysis: Forecasting and Control, $3^{\text {rd }}$ ed., Prentice Hall, Englewood Cliffs, NJ., 89-99.

Box, G. E. P., Jenkins, G. M., \& Reinsel, G. C. (1994) Time Series Analysis: Forecasting and Control, $3^{\text {rd }}$ ed., Prentice Hall, Englewood Cliffs, NJ., 224-247.

Brockwell, P. J., \& Davis, R. A. (1996). Introduction to Time Series and Forecasting., Springer, New York., Sections 3.3 and 8.3.

Dickey, D. A., \& Fuller, W. A. (1979) Distribution and the Estimators for Autoregressive Time Series With a Unit Root., Journal of the American Statistical Association, Vol. 74, No. 366, 427-431.

Dickey, D. A., Hasza, D. P., \& Fuller, W. A. (1984). Testing for Unit Roots in Seasonal Time Series., Journal of the American Statistical Association, Vol. 79, No. 386, 355367.
Durbin, J., \& Koopman, S. J. (2001). Time Series Analysis by State Space Methods., Oxford University Press.

Gardner, G., Harvey, A. C., \& Phillips, G. D. A. (1980). Algorithm AS154. An algorithm for exact maximum likelihood estimation of autoregressive-moving average models by means of Kalman filtering., Applied Statistics, 29, 311-322.

Harvey, A. C. (1993). Time Series Models, $2^{\text {nd }}$ Edition, Harvester Wheatsheaf., sections 3.3 and 4.4 .

Jones, R. H. (1980). Maximum likelihood fitting of ARMA models to time series with missing observations., Technometrics, 20, 389-395.

Kwiatkowski, D., Phillips, P. C. B., Schmidt, P., \& Shin, Y. (1992). Testing the Null Hypothesis of Stationarity against the Alternative of a Unit Root., Journal of Econometrics, 54, 159-178.

Rogers, A. J. (1986). Modified Lagrange Multiplier Tests for Problems with One-Sided Alternatives, Journal of Econometrics, NorthHolland., 31, 341-361.

Said, S. E., \& Dickey, D. A. (1984) Testing for Unit Roots in AutoregressiveMoving Average Models of Unknown Order., Biometrika, 71, 599-607.

Sakamoto, Y., Ishiguro, M., \& Kitagawa, G. (1986). Akaike Information Criterion Statistics., D. Reidel Publishing Company.

Shumway, R. H., \& Stoffer, D. S. (2006). Time Series Analysis and Its Applications: with R Examples, $2^{\text {nd }}$ ed., Springer, New York.

Tsokos, C. P. (1973). Forecasting Models from Non-Stationary Time Series-Short Term Predictability of Stocks., Mathematical Methods in Investment and Finance., North Holland Publishing Co., 520-63.

Wei, W. W. S. (2006). Time Series Analysis: Univariate and Multivariate Methods, $2^{\text {nd }}$ ed., Pearson Education, Inc. 